ISSN 2072-6643

www.mdpi.com/journal/nutrients

Review

\title{
Perceived Barriers to Application of Glycaemic Index: Valid Concerns or Lost in Translation?
}

\author{
Shannan M. Grant ${ }^{1} *$ and Thomas M. S. Wolever ${ }^{1,2}$
}

1 Department of Nutritional Sciences, University of Toronto, 150 College Street, Toronto, Ontario, M5S 3E2, Canada

2 Division of Endocrinology and Metabolism, Department of Medicine, St. Michael's Hospital, 61 Queen Street East, Toronto, Ontario, M5C 2T2, Canada; E-Mail: thomas.wolever@utoronto.ca

* Author to whom correspondence should be addressed; E-Mail: shannan.grant@ utoronto.ca; Tel.: +1-416-978-0548.

Received: 10 January 2011; in revised form: 17 February 2011 / Accepted: 23 February 2011 / Published: 28 February 2011

\begin{abstract}
The term glycaemic-index (GI) originally appeared in the literature in the early 1980s. GI categorizes carbohydrate according to glycaemic effect postprandially. Since its inception, GI has obtained and maintained interest of academics and clinicians globally. Upon review of GI literature, it becomes clear that the clinical utility of GI is a source of controversy. Can and should GI be applied clinically? There are academics and clinicians on both sides of the argument. Certainly, this controversy has been a stimulus for the evolution of GI methodology and application research, but may also negatively impact clinicians' perception of GI if misunderstood. This article reviews two assessments of GI that are often listed as barriers to application; the GI concept is (1) too complex and (2) too difficult for clients to apply. The literature reviewed does not support the majority of purported barriers, but does indicate that there is a call from clinicians for more and improved GI education tools and clinician GI education. The literature indicates that the Registered Dietitian (RD) can play a key role in GI knowledge translation; from research to application. Research is warranted to assess GI education tool and knowledge needs of clinicians and the clients they serve.
\end{abstract}

Keywords: glycaemic-index; knowledge-translation; education; clinician; dietitian; barrier(s) 


\section{Introduction}

Glycaemic-index (GI) first appeared in the literature in the early 1980s as a means by which to categorize carbohydrate according to glycaemic effect postprandially [1,2]. Carbohydrate containing foods can be categorized according to the following GI classes: low-GI (<55); medium GI (55 to 69) and high GI $(\geq 70)$ [3]. The GI is based upon a glucose (reference) scale where glucose has a GI of 100 (using standardized methodology). For instance, the high GI cut point can be expressed as 70/100 [4]. Postprandially, starchy foods included in the low-GI category are absorbed more slowly across the intestine than medium or high GI foods. Slow postprandial intestinal absorption of starchy low-GI food results in a gradual increase in blood glucose (BG) and lower peak BG when compared to the prominent peak in BG observed after consuming a high GI food [2,4,5]. A large body of data suggests a diet composed of low-GI food has a role to play in the prevention or treatment of a number of chronic conditions including type 2 diabetes mellitus (T2DM), cardiovascular disease, and cancer [6-12]. Despite the existence of these supporting data, the utility of GI continues to be a topic of debate.

Clinicians, more specifically Registered Dietitians (RD), continue to question the utility of the GI concept in their practice [13]. A postal cross-sectional survey was conducted in Canada (2003), including members of the Dietitians of Canada (DC) and Ordre Professionnel des Dietetistes du Quebec (OPDQ). This questionnaire was created to assess how many RDs identify as "users" or "non-users" of the GI concept, what RDs perceive to be benefits of and barriers to GI utility, RDs general knowledge of GI, and their confidence in teaching the concept [13]. A total of $6060 \mathrm{RDs}$ $(\mathrm{DC}=4014 ;$ OPDQ $=2046)$ were originally contacted. Of the total respondents $(n=2857)$, $40 \%(n=724)$ identified as users, while 60\% $(n=1,081)$ identified as non-users. One thousand and fifty-seven respondents reported treating patients with diabetes mellitus (DM). Of this subset, $39 \%(n=415)$ identified as users and 61\% $(n=642)$ as non-users. Only 3\% of respondents $(n=642)$ were unaware of the GI concept. Of members who identified as users, $90 \%$ used GI as a general descriptor of carbohydrate absorption (fast [high GI] versus slow [low-GI]) and 56\% reported teaching GI with the aim of facilitating glycaemic control. The following three barriers were most commonly selected by non-users working with clients living with DM: (1) $57 \%$ felt the GI concept was too complex for patients to understand, (2) $46 \%$ reported lack of education resources, (3) $31 \%$ did not know how to use the concept. Of the study participants, those who identified as users were more likely to have a greater DM caseload and perceive benefits to and confidence in teaching the GI concept.

\section{GI Knowledge Translation}

Knowledge translation is a term used by health researchers to describe the relationship between knowledge creation and application [14]. The translation of knowledge discovery into practice applications or policy is a fundamental component of the knowledge-to-action process. In the current research environment, agencies supporting health research do not only expect research be published in peer-reviewed journals, but also that plans will be outlined, within the original grant proposal, to translate the research findings into practice or policy [14]. A rather new concept among researchers, this concept may then inspire the question, "who should take responsibility for the end use of findings?"[15]. 
As outlined in the literature, clinicians, specifically RDs, can play a key role in facilitating GI education [13,15-18]. It is therefore important that RDs perceived barriers to GI utility be addressed by researchers. Studies have shown that RDs that work with people living with $\mathrm{DM} \geq 11$ years are more likely to use the GI concept $[13,19]$. Research has also shown that outpatient RDs are more likely than in-patient clinicians to use GI education [13]. Research looking at why these differences exist, whether or not perceived barriers to GI utility are valid, and how to overcome these barriers are key to facilitating GI knowledge translation and continuing the knowledge-to-action cycle.

\section{Perceived Barriers to Knowledge Translation}

The perceived barriers to GI utility highlighted in Kalergis et al. (2006) are recurrently noted by GI critics within and outside of Canada [13,19-22]. Inspired by these works, this article will review the three aforementioned barriers along with other commonly documented barriers to GI application. Many of these barriers have been used as justification for the assessment that GI is too difficult for clinicians and/or nutrition professionals to teach and is too difficult for clients to understand and apply. The reviewed literature will, therefore, be organized under the following headings/themes: (1) The GI concept is too complex (for RDs to teach and clients to understand) and (2) The GI concept is too difficult for clients to apply. The following paragraphs will show that perceived barriers to GI utility are, in many cases, unfounded or easily addressed. Despite this, these criticisms are ever-present in peer-reviewed literature and popular media; literature clinicians utilize to make professional judgements.

\subsection{Assessment 1: The GI Concept Is Too Complex (for RDs to Teach and Clients to Understand)}

The perceived complexity of GI has been repeatedly noted as a barrier to GI utility. The assessment that the GI concept is too complex is often supported by the following three barriers or criticisms: GI education opposes current dietary guidelines (and is therefore confusing), GI terminology is confusing and there is a shortage of GI education materials [13,19-22]. This section will examine these criticisms using available literature.

The Canadian Diabetes Association (CDA) (2008) (Clinical Practice Guidelines) recommends that GI education be used as a supplement to standard care with use varying by client interest, ability and need. GI education, therefore, should be presented as a supplement to dietary recommendations; not as an alternative to it [23]. Current North American dietary recommendations include suggestions regarding serving/portion size (varying by sex and stage of life), fibre, fat and sugar intake and promotes dietary variation/diversity and moderation. GI education focuses primarily on the concept of carbohydrate quality and glycaemic response [1,2,24]. Despite the existence and availability of this information, GI critics seem confident that the low-GI diet encourages increased use of foods high in fat and sugar and promotes an increase in energy consumption [20,22]. The existence of this misinformation is not surprising, especially in light of the recent finding that only $61 \%$ of Canadian RDs who identify as GI education users and $26 \%$ of non-users report being aware of CDAs position on GI [13]. A number of studies display that low-GI foods can be consumed as part of a diet based on current dietary recommendations. For instance, Grant et al. [25] used low-GI education as a supplement to standard care to obtain improved glycaemic control in women with gestational diabetes mellitus and impaired glucose tolerance. Addition of low-GI education did not result in divergence 
from current dietary recommendations in this group; energy and macronutrient intake was matched to controls post intervention. Moreover, Frost et al. [26] facilitated adherence to current dietary recommendations using low-GI education. In this study, participants on the low-GI diet consumed less dietary fat and more fibre. Similar results have been found by others [27-30].

To encourage efficient GI knowledge translation, clinicians and academics must work together to efficiently translate scientific jargon and/or concepts. This involves establishing appropriate translations and audience-appropriate phraseology; whether the audience is composed of researchers and clinicians, individual clients or whole populations [31-35]. Slabber [36] offered examples of such translations accompanied by client-focused education tools and phraseology, concluding that GI terminology need not be any more difficult than teaching other concepts included in standard medical nutrition therapy. For instance, low and high GI can be explained using terms like, "slow and fast acting carbohydrate". "Retrogradation" can be explained using the following phrasing: "When cooked (red) potatoes are cooled in the fridge, the starch in them becomes sticky and gel-like." Notwithstanding, Mendes et al. [19] found that GI was not deemed appropriate for clinical use by $78 \%$ of American RDs treating children for obesity. RDs in this study indicated they felt knowledgeable about GI $(77 \% ; n=92)$, but felt GI terminology/concepts were too challenging for study participants. Although these data were published after the publication of the following statement of the American Diabetes Association (ADA) (2005): “...use of GI can provide additional benefit over that observed when total carbohydrate is considered alone...”, American health agencies traditionally were in opposition to GI utility $[21,37,38]$. This traditional position most likely affected the sample's (American RDs') perception of GI application. Conversely, Frost et al. [26] showed that people can successfully and significantly lower diet-GI after verbal and written communication. Similar success has been noted by others [28,39].

Canadian RDs believe a shortage of GI education materials is a barrier to GI utility [13]. Clinicians and/or researchers have set out to address this resource-gap in an attempt to overcome this barrier to utility. For instance, the Canadian Diabetes Association (CDA) has published a Glycaemic-Index Education Tool that can be downloaded off the CDA website [3]. The CDA education tool includes substitution lists for low, medium and high GI food choices frequently used in the Canadian diet. The following GI categories are included in this tool: Low-GI (55 or less; choose most often), medium GI (56-69; choose more often) and high GI (70 or more; choose less often). The CDA tool format is based upon recommendations from clinical scientists, basic scientists and diabetes educators of how to best provide GI information to clients. This tool also summarizes current dietary recommendations and key take-home messages for DM management; demonstrating GI as a supplement to standard care. Variations of the low-GI food substitution list are available in peer-reviewed literature, popular media, and online [40-44]. Food substitution lists have been used in our laboratory and others to achieve a moderate difference in food choice and to obtain a significant decrease of dietary GI [45,46]. Although a very useful tool, the CDA GI education tool only lists 12-18 foods under each heading and does not include a section under each GI category for the RD to add individualized food substitutions.

American and South African RDs report a lack of usable/suitable GI teaching tools as a barrier to GI utility; rather than a lack of tools [18,19]. Understanding the Glycaemic Index is a Canadian GI education tool that illustrates that similar findings may be obtained in Canada [47]. This tool, developed by clinicians and researchers, has been published on the Canadian Sugar Institute website 
and was mailed out to RDs across Canada. Although this tool contains some very useful information, there are conceptual errors on this tool and therefore misinformation. For instance, the following statement is included in this tool "Fat or protein eaten along with carbohydrate... reduces the GI of the carbohydrate". This statement is incorrect and reflects back to the aforementioned GI myth that low-GI education encourages increased use of fat. The authors of this tool have mistakenly used the term glycaemic index when they are describing glycaemic response; highlighting and propagating a hiccup in the knowledge translation process. GI is a characteristic of available carbohydrate and is not synonymous with glycaemic response [2,4]. Protein and fat have effects on glycaemic response which are independent of those produced by carbohydrates and occur by different mechanisms $[4,48,49]$. The proper terminology would be, "Adding fat and protein to a carbohydrate reduced the glycaemic response". This education tool provides an example of how confusion, secondary to GI terminology and phraseology, may exist among clinicians and/or researchers. Research assessing clinicians' comprehension of GI concepts and terminology and examining GI misinformation in academic literature, websites and popular media are warranted.

\subsection{Assessment 2: The GI Concept Is Too Difficult for Clients to Apply}

There have been a number of barriers cited in the literature to support the assessment that the GI concept is difficult for clients to apply. Two examples of these proposed barriers include: The low-GI diet limits food choice and GI is not accepted by clients. Although noteworthy barriers to any dietary change these barriers are not currently supported by the existing data on GI. The following paragraphs will review these data.

It is unrealistic for a clinician to expect clients to consume low-GI carbohydrate $100 \%$ of the time $[36,43,50]$. The literature has identified this expectation as a limitation and an example of how low-GI limits food choice $[20,21,38]$. Conversely, the literature indicates that GI "users" recognize that medium to high GI foods may be appropriate in some cases and flexibility is important for sustainable lifestyle change [26,27,43,50-53]. Moreover, research shows one need only consume low-GI choices $50-60 \%$ of the time to obtain a significant reduction in dietary GI ( 7 to 11 units) and/or documented benefits [4,50]. Despite this, the following two barriers to low-GI diet compliance were noted by Brekke et al. [54]: lack of choice when dining out and lack of ideas when cooking at home. It is important to note, however, that this criticism is not unique to the low-GI diet. Similar barriers have been noted during dietary interventions that do not use GI education [52-56]. In general, it is challenging for one to change lifestyle behaviours from initiation to maintenance $[52,53,57,58]$. To address these concerns, clinical scientists (including RDs) in our laboratory and others have designed culturally sensitive GI recipe booklets, GI food lists and tips for dining out that outline low, medium and high GI foods [3,25,36,40,43].

The question of whether or not low-GI is accepted by clients has fuelled much research and is ever-present in GI literature. Once a clinician makes the judgement call that the literature supporting a dietary intervention is adequate, he/she often then asks the questions, "will clients eat the recommended food?" and "is this lifestyle change sustainable?". There is a wealth of literature that indicates the answer to both questions is yes in the context of a low-GI diet. For instance, Burani and Longo [59] assessed the effect of low-GI medical-nutrition-therapy (MNT) on multi-ethnic 
American adults living with type 1 and $2 \mathrm{DM}(n=21)$ one year after education. At baseline $90 \%$ of the participants reported not understanding GI and 19\% reported feeling they could include it in their current lifestyle. Post-intervention, average dietary GI was 45 and a statistically significant decrease in GI was achieved by $95 \%$ participants. Moreover, post-intervention, $85 \%$ of participants reported they had adequate understanding of GI and 95\% felt they possessed enough knowledge to apply it in their lifestyle after the study. All study participants accepted GI education as a useful supplement to current dietary recommendations, perceived low-GI foods to be healthy and reported beneficial effects on glycaemic control and weight management. Perhaps most important to note, $100 \%$ of participants reported planning to include low-GI as a permanent lifestyle change; indicative of low-GI diet acceptability and sustainability in this sample. These findings are in agreement with those of a recent prospective randomized control trial conducted in Australia where pregnant women following a low-GI diet were more likely to agree their study diet was easier to follow in comparison to those on a medium-to-high GI diet [51,59].

Comparable results have also been seen in children and young adults [60,61]. For instance, using a cross-over design, Nansel et al. [60] looked at low-GI food acceptability of standard[S] versus low-GI[LGI] menus in a youth camp for children with type 1 and 2 DM in the United States. Food service staff were provided with low-GI food and cooking instructions aimed to facilitate creation of a low-GI menu. Camp kitchen staff reported low-GI foods were acceptable in terms of preparation effort, perceived healthiness and youth appeal. A questionnaire (Likert Scale format; 1 = "I didn't like it at all", 5 = "I liked it a whole lot") was provided to the children after every meal and evening snack. Camp attendees ( $n=140$; age 7 to 16 ) provided comparable ratings for low-GI food and standard foods served at dinner[D] and snacks[SNK] (D = 3.68 [LGI] vs. 3.79 [S], $p=0.30$; SNK = 3.74 [LGI] vs. $3.79[\mathrm{~S}], p=0.60)$. On the other hand, low-GI foods at breakfast[BFST] and lunch[L] were acceptable, but were rated lower than standard foods (BFST $=3.76$ [LGI] vs. $4.04[\mathrm{~S}], p=0.01$; $\mathrm{L}=3.64[\mathrm{LGI}]$ vs. $3.88[\mathrm{~S}], p=0.01$ ). Similar findings were obtained during a previous long term prospective randomized trial in children living with T1DM. In this study, children on a low-GI diet did not decrease dietary quality or choice in comparison to a control group using traditional carbohydrate exchange dietary advice [36,39].

\section{Glycaemic Index: Breaking down the Barriers}

In Canada, the question of whether to use or not to use GI education as part of medical nutrition therapy for diabetes prevention and treatment is left to the discretion of the clinician. Clinicians often look to colleagues, key journal articles, education tools and online media for information when deciding which therapies to utilize. It is therefore important that reliable literature and education materials/tools are easily available to keep interested practitioners well-informed. Propagation of GI mythology represents a barrier to GI application and an interruption of the scientific process. An aim of this article is to address and move beyond commonly cited GI mythology, while inspiring research and development that will test GI utility and highlight and overcome valid barriers to GI application. Clinicians, specifically Registered Dietitians (RDs), can play a key role in facilitating GI knowledge translation from laboratory to client. It is therefore important that RDs' perceived barriers to GI utility are studied and RDs be included in the research process. 
While GI research continues and data on GI-utility and/or methodology are collected, there is a need for ongoing review of perceived and actual barriers to GI utility from the perspective of the educator and the client. Many of the perceived barriers reviewed in this paper may not be currently supported by existing data, but indicate that supplementary research is warranted. Research on clinicians' and clients' perceptions, knowledge of and application of GI is still needed to ensure that clinicians have access to adequate evidence-based literature on which to base their professional opinions. Existing data also support that there is a perceived deficiency in "reliable" GI education and/or reference materials available to clinicians. Review of existing GI education tools and, in some cases, development of new GI education tools for clinicians with the input of clinicians and clients is therefore warranted.

\section{Conflict of Interests}

Thomas Wolever is the President of Glycemic Index Laboratories, Toronto, Ontario, Canada [62]; a contract research organization. Wolever is also co-author of the North American editions of a range of popular books on GI, including "The Glucose Revolution" and "The New Glucose Revolution". Shannan Grant has no conflict of interest to disclose.

\section{References}

1. Jenkins, D.J.A.; Wolever, T.M.S.; Taylor, R.H. Glycemic Index of Foods: A Physiological Basis for Carbohydrate Exchange. Am. J. Clin. Nutr. 1981, 34 (3), 362-366.

2. Wolever, T.M.S.; Jenkins, D.J.A.; Jenkins, A.L.; Josse, R.G. The Glycemic Index: Methodology and Clinical Implications. Am. J. Clin. Nutr. 1991, 54 (5), 846-854.

3. Canadian Diabetes Association (CDA). The Glycemic Index Tool. Available online: http://www.diabetes.ca/for-professionals/resources/nutrition/glycemic-index/ (accessed on 14 March 2010).

4. Wolever, T.M.S. The Glycaemic Index: A Physiological Classification of Dietary Carbohydrate; CABI: Cambridge, MA, USA, 2006.

5. Wolever, T.M.S. Physiological Mechanisms and Observed Health Impacts Related to the Glycaemic Index: Some Observations. Int. J. Obes. 2006, 30 (Suppl. 3), S72-S78.

6. Augustin, L.S.A.; Gallus, S.; Franceschi, S.; Negri, E.; Jenkins, D.J.A.; Kendall, C.W.C.; Dal Maso, L.; Talamini, R.; La Vecchia, C. Glycemic Index and Load and Risk of Upper Aero-digestive Tract Neoplasms (Italy). Cancer Causes Control 2003, 14 (7), 657-662.

7. Augustin, L.S.A.; Polesel, J.; Bosetti, C.; Kendall, C.W.C.; La Vecchia, C.; Parpinel, M.; Conti, E.; Montella, M.; Franceschi, S.; Jenkins, D.J.A.; Dal Maso, L. Dietary Glycemic Index, Glycemic Load and Ovarian Cancer Risk: A Case-control Study in Italy. Ann. Oncol. 2003, 14 (1), $78-84$.

8. Tavani, A.; Bosetti, C.; Negri, E.; Augustin, L.S.; Jenkins, D.J.A.; La Vecchia, C. Carbohydrates, Dietary Glycaemic Load and Glycaemic Index, and Risk of Acute Myocardial Infarction. Heart 2003, 89 (7), 722-726.

9. Augustin, L.S.A.; Dal Maso, L.; Vecchia, C.L.; Parpinel, M.; Negri, E.; Vaccarella, S.; Kendall, C.W.C.; Jenkins, D.J.A.; Franceschi, S. Dietary Glycemic Index and Glycemic Load, and Breast Cancer Risk: A Case-control Study. Ann. Oncol. 2001, 12 (11), 1533-1538. 
10. Franceschi, S.; Dal Maso, L.; Augustin, L.; Negri, E.; Parpinel, M.; Boyle, P.; Jenkins, D.J.A.; La Vecchia, C. Dietary Glycemic Load and Colorectal Cancer Risk. Ann. Oncol. 2001, 12 (2), 173-178.

11. Tsai, C.; Leitzmann, M.F.; Willett, W.C.; Giovannucci, E.L. Glycemic Load, Glycemic Index, and Carbohydrate Intake in Relation to Risk of Cholecystectomy in Women. Gastroenterology 2005, 129 (1), 105-112.

12. Tsai, C.; Leitzmann, M.F.; Willett, W.C.; Giovannucci, E.L. Dietary Carbohydrates and Glycaemic Load and the Incidence of Symptomatic Gall Stone Disease in Men. Gut 2005, 54 (6), 823-828.

13. Kalergis, M.; Pytka, E.; Yale, J.; Mayo, N.; Strychar, I. Canadian Dietitians' Use and Perceptions of Glycemic Index in Diabetes Management. Can. J. Diet. Pract. Res. 2006, 67 (1), 21-27.

14. Canadian Institutes of Health Research. About Knowledge Translation, 2009. Available online: http://www.cihr-irsc.gc.ca/e/29418.html (accessed on 15 April 2010).

15. Atkinson, S.A. A Nutrition Odyssey: Knowledge Discovery, Translation, and Outreach: 2006 Ryley-Teffs Memorial Lecture. Can. J. Diet. Pract. Res. 2006, 67 (3), 150-156.

16. Venters, J.Y.; Hunt, A.E.; Pope, J.F.; Molaison, E.F. Are Patients with Diabetes Receiving the Same Message from Dietitians and Nurses? Diabetes Educ. 2004, 30 (2), 293-300.

17. Heller, T.; Maislos, M.; Shahar, D. Physicians' and Nurses' Knowledge and Attitude Towards Nutritional Therapy in Diabetes. Harefuah 2007, 146 (9), 670-674.

18. Vorster, H.H.; Banitz, S.A.; Carter, D.S.; Cuba, Z.; Dekeda, T.; Dolman, R.; Doubell, M.; Erasmus, C.J.L.; Faber, A.; Fivaz, C.; et al. The Glycaemic Index in Practice-Consensus Statement of a Small Group of South African Dietitians. South Afr. J. Clin. Nutr. 2005, 18 (3), 260-264.

19. Mendes, K.; Iselin, J.; Edelstein, S. Dietitians' Use of the Glycemic Index/Glycemic Load as a Counseling Tool for Overweight Children. Top. Clin. Nutr. 2006, 21 (4), 300-311.

20. Beebe, C. Diets With Low Glycemic Index: Not Ready for Practice Yet! Nutr. Today 1999, $34(2), 82-86$.

21. Franz, M.J. In Defense of the American Diabetes Associations Recommendations on the Glycemic Index. Nutr. Today 1999, 34 (2), 78-81.

22. Pi-Sunyer, F.X. Glycemic Index and Disease. Am. J. Clin. Nutr. 2002, 76 (1), 290S-298S.

23. Canadian Diabetes Association. Canadian Diabetes Association 2008 Clinical Practice Guidelines for the Prevention and Management of Diabetes in Canada. Can. J. Diabetes 2008, 32 (Suppl. 1), S1-S201.

24. Wolever, T.M.S.; Jenkins, D.J.A.; Vuksan, V.; Jenkins, A.L.; Buckley, G.C.; Wong, G.S.; Josse, R.G. Beneficial Effect of a Low Glycaemic Index Diet in Type 2 Diabetes. Diabet. Med. 1992, 9 (5), 451-458.

25. Grant, S.M.; Wolever, T.M.S.; O’Connor, D.L.; Nisenbaum, R.; Josse, R.G. Effect of a Low Glycaemic Index Diet on Blood Glucose in Women with Gestational Hyperglycaemia. Diabetes Res. Clin. Pract. 2011, 91 (1), 15-22.

26. Frost, G.; Wilding, J.; Beecham, J. Dietary Advice Based on the Glycaemic Index Improves Dietary Profile and Metabolic Control in Type 2 Diabetic Patients. Diabet. Med. 1994, 11 (4), 397-401. 
27. Frost, G.; Keogh, B.; Smith, D.; Akinsanya, K.; Leeds, A. The Effect of Low-glycemic Carbohydrate on Insulin and Glucose Response in Vivo and in Vitro in Patients with Coronary Heart Disease. Metab. Clin. Exp. 1996, 45 (6), 669-672.

28. Brand-Miller, J.; Hayne, S.; Petocz, P.; Colagiuri, S. Low-glycemic Index Diets in the Management of Diabetes: A Meta-analysis of Randomized Controlled Trials. Diabetes Care 2003, 26 (8), 2261-2267.

29. Schulz, M.; Liese, A.D.; Mayer-Davis, E.; D’Agostino, R.B., Jr.; Fang, F.; Sparks, K.C.; Wolever, T.M.S. Nutritional Correlates of Dietary Glycaemic Index: New Aspects From a Population Perspective. Br. J. Nutr. 2005, 94, 397-406.

30. Miller, C.K.; Davis-Gutshcall, M.; Mitchell, D.C. Change in food choices following a glycemic load intervention in adults with type 2 diabetes. J. Am. Diet. Assoc. 2009, 109, 319-324.

31. Reach, G. Linguistic Barriers in Diabetes Care. Diabetologia 2009, 52 (8), 1461-1463.

32. Tokuda, Y.; Okamoto, S.; Yoshioka, Y.; Aizawa, M.; Tanaka, M.; Motomura, K.; Hayano, K. The Influence of Medical Jargon Mixed with Foreign Terminology in the Japanese Clinical Environment. Intern. Med. 2008, 47 (14), 1329-1334.

33. Singh, H.; Duerksen, D.R. Survey of Clinical Nutrition Practices of Canadian Gastroenterologists. Can. J. Gastroenterol. 2006, 20 (8), 527-530.

34. van Mook, W.N.K.A.; van Luijk, S.J.; O’Sullivan, H.; Wass, V.; Harm Zwaveling, J.; Schuwirth, L.W.; van der Vleuten, C.P.M. The Concepts of Professionalism and Professional Behaviour: Conflicts in Both Definition and Learning Outcomes. Eur. J. Intern. Med. 2009, 20 (4), e85-e89.

35. Deuster, L.; Christopher, S.; Donovan, J.; Farrell, M. A Method to Quantify Residents' Jargon Use during Counseling of Standardized Patients about Cancer Screening. J. Gen. Intern. Med. 2008, 23 (12), 1947-1952.

36. Slabber, M. Complexities of Consumer Understanding of the Glycaemic Index Concept and Practical Guidelines for Incorporation in Diets. South Afr. J. Clin. Nutr. 2005, 18 (3), 252-257.

37. American Diabetes Association. American Diabetes Association: Clinical Practice Recommendations 2005. Introduction. Diabetes Care 2005, 28 (Suppl. 1), S1-S2.

38. Franz, M.J.; Bantle, J.; Beebe, C.A.; Brunzell, J.D.; Chiasson, J.; Garg, A.; Holzmeister, L.A.; Hoogwerf, B.; Mayer-Davis, E.; Mooradian, A.; Purnell, J.Q.; Wheeler, M. American Diabetes Association Position Statement: Evidence-based Nutrition Principles and Recommendations for the Treatment and Prevention of Diabetes and Related Complications. J. Am. Diet. Assoc. 2002, 102 (1), 109-118.

39. Gilbertson, H.R.; Thorburn, A.W.; Brand-Miller, J.C.; Chondros, P.; Werther, G.A. Effect of low-glycemic-index dietary advice on dietary quality and food choice in children with type 1 diabetes. Am. J. Clin. Nutr. 2003, 77 (1), 83-90.

40. The University of Sydney. The Glycemic Index, 2010. Available online: http://www.glycemicindex.com/ (accessed on 10 November 2010).

41. Atkinson, F.; Chan, S.; Louie, J. GI values list of common Asian foods, 2009. Available online: http://www.ginewschi.blogspot.com (accessed on 14 April 2010).

42. Atkinson, F.S.; Foster-Powell, K.; Brand-Miller, J.C. International Tables of Glycemic Index and Glycemic Load Values: 2008. Diabetes Care 2008, 31 (12), 2281-2283. 
43. Brand-Miller, J.C.; Foster-Powell, K. Diets with Low Glycemic Index: From Theory to Practice. Nutr. Today 1999, 34 (2), 64-72.

44. Foster-Powell, K.; Holt, S.H.A.; Brand-Miller, J.C. International Table of Gylcemic Index and Glycemic Load Values: 2002. Am. J. Clin. Nutr. 2002, 76 (1), 5-56.

45. Wolever, T.M.S.; Gibbs, A.L.; Mehling, C.; Chiasson, J.; Connelly, P.W.; Josse, R.G.; Leiter, L.A.; Maheux, P.; Rabasa-Lhoret, R.; Rodger, N.W.; Ryan, E.A. The Canadian Trial of Carbohydrates in Diabetes (CCD), A 1-y Controlled Trial of Low-glycemic-index Dietary Carbohydrate in Type 2 Diabetes: No Effect on Glycated Hemoglobin but Reduction in C-reactive Protein. Am. J. Clin. Nutr. 2008, 87 (1), 114-125.

46. Heilbronn, L.K.; Noakes, M.; Clifton, P.M. Effect of Energy Restriction, Weight Loss, and Diet Composition on Plasma Lipids and Glucose in Patients with Type 2 Diabetes. Diabetes Care 1999, 22 (6), 889-895.

47. Canadian Sugar Institute. Clips of Sugars: Understanding Glycemic Index, 2008. Available online: http://www.sugar.ca/english/healthprofessionals/diabetes.cfm (accessed on 4 April 2010).

48. Wolever, T.M.S. Glycemic Index versus Glycemic Response: Nonsynonymous Terms. Diabetes Care 1992, 15 (10), 1436-1437.

49. Wolever, T.M.S.; Jenkins, D.J.A.; Vuksan, V.; Katzman, L.; Jenkins, A.L.; Josse, R.G. Variation in Meal Fat Content Does Not Affect the Relative Glycaemic Response of Spaghetti in Subjects with Type II Diabetes. Diabetes Nutr. Metab. 1992, 5 (3), 191-197.

50. Brand-Miller, J.C. Importance of Glycemic Index in Diabetes. Am. J. Clin. Nutr. 1994, 59 (Suppl. 3), 747S-752S.

51. Moses, R.G.; Luebcke, M.; Davis, W.S.; Coleman, K.J.; Tapsell, L.C.; Petocz, P.; Brand-Miller, J.C. Effect of a Low-glycemic-index Diet During Pregnancy on Obstetric Outcomes. Am. J. Clin. Nutr. 2006, 84 (4), 807-812.

52. Radakovich, K.; Heilbrun, L.K.; Venkatranamamoorthy, R.; Lababidi, S.; Klurfeld, D.M.; Djuric, Z. Women Participating in a Dietary Intervention Trial Maintain Dietary Changes Without Much Effect on Household Members. Nutr. Cancer 2006, 55 (1), 44-52.

53. Shai, I.; Schwarzfuchs, D.; Henkin, Y.; Shahar, D.R.; Witkow, S.; Greenberg, I.; Golan, R.; Fraser, D.; Bolotin, A.; Vardi, H. Weight loss with a low-carbohydrate, Mediterranean, or low-fat diet. N. Engl. J. Med. 2008, 359 (3), 229-241.

54. Brekke, H.K.; Sunesson, Å.; Axelsen, M.; Lenner, R.A. Attitudes and Barriers to Dietary Advice Aimed at Reducing Risk of Type 2 Diabetes in First-degree Relatives of Patients with Type 2 Diabetes. J. Hum. Nutr. Diet. 2004, 17 (6), 513-521.

55. Galasso, P.; Amend, A.; Melkus, G.D.; Nelson, G.T. Barriers to Medical Nutrition Therapy in Black Women with Type 2 Diabetes Mellitus. Diabetes Educ. 2005, 31 (5), 719-725.

56. Gutschall, M.D.; Miller, C.K.; Mitchell, D.C.; Lawrence, F.R. A Randomized Behavioural Trial Targeting Glycaemic Index Improves Dietary, Weight and Metabolic Outcomes in Patients with Type 2 Diabetes. Public Health Nutr. 2009, 12 (10), 1846-1854.

57. Steg, P.G.; Verdier, J.; Carré, F.; Darne, B.; Ducardonnet, A.; Julliene, G.; Farnier, M.; Giral, P.; Haï, R. A Randomised Trial of Three Counselling Strategies for Lifestyle Changes in Patients with Hypercholesterolemia Treated with Ezetimibe on Top of Statin Therapy (TWICE). Arch. Cardiovasc. Dis. 2008, 101 (11-12), 723-735. 
58. O’Hea, E.L.; Boudreaux, E.D.; Jeffries, S.K.; Carmack Taylor, C.L.; Scarinci, I.C.; Brantley, P.J. Stage of Change Movement Across Three Health Behaviors: The Role of Self-efficacy. Am. J. Health Promot. 2004, 19 (2), 94-102.

59. Burani, J.; Longo, P.J. Low-glycemic Index Carbohydrates: An Effective Behavioral Change for Glycemic Control and Weight Management in Patients with Type 1 and 2 Diabetes. Diabetes Educ. 2006, 32 (1), 78-88.

60. Nansel, T.R.; Gellar, L.; Zeitzoff, L. Acceptability of Lower Glycemic Index Foods in the Diabetes Camp Setting. J. Nutr. Educ. Behav. 2006, 38 (3), 143-150.

61. Miller, C.K.; Lindberg, D.V. Evaluation of a Computer-based Game About the Glycemic Index Among College-aged Students. Top. Clin. Nutr. 2007, 22 (3), 299-306.

62. Glycemic Index Laboratories. Available online: http://www.gilabs.com/main/index.php (accessed on 26 February 2011).

(C) 2011 by the authors; licensee MDPI, Basel, Switzerland. This article is an open access article distributed under the terms and conditions of the Creative Commons Attribution license (http://creativecommons.org/licenses/by/3.0/). 